\title{
Air Pollution Mix and Emergency Room Visits for Respiratory and Cardiac Diseases in Taipei
}

\author{
Jing-Shiang Hwang ${ }^{1}$, Tsuey-Hwa $\mathrm{Hu}^{1}$ and Chang-Chuan $\mathrm{Chan}^{2}$ \\ 1 Academia Sinica \\ ${ }^{2}$ National Taiwan University
}

\begin{abstract}
To clarify the contribution of ambient air pollutants to acute health effects, we examined the association between daily air pollution levels and emergency room (ER) visits for respiratory and cardiac diseases in Taipei City, Taiwan from January 1997 to December 1998. Average daily concentrations of particulate matter less than $2.5 \mu \mathrm{m}$ in aerodynamic diameter $\left(\mathrm{PM}_{2.5}\right), \mathrm{PM}_{10}$, carbon monoxide, sulfur dioxide, nitrogen dioxide and ozone were obtained from ambient air quality monitoring stations. The daily counts of ER visits stratified by diagnosis and age were modeled by both single-pollutant and multi-pollutant Poisson regression models with adjustment of confounding factors to evaluate the effects of individual pollutants. A mixture model was constructed by adding ratios of the pollutants to the multi-pollutant model to examine the air pollution mixture on ER visits. The single-pollutant models showed that an interquartile range change of $\mathrm{PM}_{2.5}\left(16 \mu \mathrm{g} / \mathrm{m}^{3}\right)$ was associated with increased ER visits for respiratory disease in all age groups, with relative risks 1.04 to 1.06 and increased ER visits for cardiac disease in adult and elderly age groups, with a relative risk of 1.05. Gaseous pollutants, mainly $\mathrm{NO}_{2}$ and $\mathrm{CO}$, were also associated with increased visits by children for respiratory disease and visits by adults and elderly individuals for cardiac disease. Examination of joint effect of mixes of $\mathrm{PM}_{2.5}$ and gaseous pollutants showed that an interquartile range increase was associated with increases in ER visits by children for respiratory disease and by adults for cardiac disease, with a relative risk of 1.09 .
\end{abstract}

Key words: Air pollution, cardiac disease, emergency room visits, respiratory disease. 


\section{Introduction}

Epidemiologic studies conducted in cities around the world during the past decade have reported significant associations between air pollution and increased mortality and hospital admissions due to respiratory and cardiovascular diseases (Schwartz 1996, 1997, 1999, Schwartz and Morris 1995, Schwartz et al. 2000, Burnett et al. 1995, 1997, 2001, Linn et al. 2000, Zhang et al. 2002). Some studies have also examined the effects of air pollution on emergency room (ER) visit statistics, which are expected to be a more sensitive indicator of acute health effects from air pollution than hospital admission data for a variety of reasons. First, whereas only a subset of patients visiting the ER is hospitalized, those that are more critically ill, ER visit records also include patients with mild and moderate conditions, who may not require hospitalization. In addition, in contrast to hospital admissions, which may not occur for several days after the onset of symptoms, ER visits more closely reflect acute response to changes in air quality during a particular time period.

Although ER visit statistics have been used in a number of studies, the scope of these studies has generally been limited to specific conditions such as asthma and/or specific subpopulations, due primarily to limitations in the availability of ER data (Delfino et al. 1998, Lipsett et al. 1997, Xu et al. 1995, Sunyer et al. 1993, Norris et al. 1999). Since most countries lack a standardized system for medical surveillance, the acquisition and categorization of data from emergency department patient records can be costly and cumbersome. In the United States, for example, national statistics on injuries and infectious are being increasingly monitored by various agencies, but no centralized system is yet available despite ongoing efforts to standardize data collection practices.

To overcome these limitations, the authors have selected data from a unique resource, the National Health Insurance database in Taipei, Taiwan to study the association between air pollution and ER visits. The National Health Insurance database is a centralized collection of detailed medical information for 2.7 million people, including visits to emergency rooms in major medical centers and small clinics in Taipei. The database provide a unique opportunity to study the effects of air pollution on daily ER due to general cardiorespiratory disease in a city with a large population, which may be applicable to other densely populated cities.

The evidence of health effects of air pollution provided by these studies is mainly based on the associations between single outcome and single air pollutant. In order to reflect the fact that air pollutants always occurred as a mixture, multi-pollutant models have been used to estimate the effect of one pollutant by adjusting other pollutants or the additive effects of significant pollutants found in the single-pollutant models (Burnett et al. 1997, Sheppard et al. 1999, Mool- 
gavkar et al. 1997, Wong et al. 2002). These multi-pollutant models usually include multiple pollutants as additive independent variables in the regression. Few studies have discussed that the joint effects of pollutants may be affected by the interaction of the pollutant variables (Hwang and Chen 1999). No previous studies have ever treated air pollution variables as the pollution mix of several air pollutants together in their multi-pollutant models. Since air pollutants seldom change their concentrations at a fixed proportion concurrently in the environment, we expected their combined effects to be affected by both total amounts and proportions in the pollution mix. In order to reflect real air pollution situations in the environment, we proposed to construct a mixture model by adding ratios of two pollutant levels into the multi-pollutant model. These ratio terms are formed to measure the blending effect of the pollutants in the air pollution mix. This less variant ratio will have no effect on the response if the two pollutants are highly correlated. In this case, we expect no significant difference in the effect between multi-pollutant and mixture models. In a real environment, the ratio between gaseous pollutants and particulate matter usually varies daily because of various particulate matter emitting sources. Therefore, the authors believe the mixture models proposed in this paper can better clarify the contribution of air pollution as a whole to acute health effects than most previous multi-pollutant models.

In this study, we examined the health effects of air pollution mix on the daily ER visits for respiratory and cardiac diseases in Taipei City from 1997 to 1998. The pollutant mixtures evaluated in this study included fine particles (particulate matter with an aerodynamic diameter less than $\left.2.5 \mu \mathrm{m}, \mathrm{PM}_{2.5}\right), \mathrm{PM}_{10}$, carbon monoxide $(\mathrm{CO})$, sulfur dioxide $\left(\mathrm{SO}_{2}\right)$, nitrogen dioxide $\left(\mathrm{NO}_{2}\right)$ and ozone $\left(\mathrm{O}_{3}\right)$. Single-pollutant lagged Poisson regression models were first applied to examine the association between individual air pollutant's daily concentration fluctuation and daily changes in ER visit counts, after adjusting for temporal and seasonal patterns, day of the week and weather factors. The air pollution mix effects on the relative risks of ER visits for respiratory and cardiac diseases in three age groups were examined by both multi-pollutant and the proposed mixture models for comparison.

\section{Materials and Methods}

\subsection{Emergency room visits}

The Bureau of National Health Insurance (BNHI) collected computerized records of daily clinic visits for all contracted medical institutions which have covered medical services of more than $96 \%$ of the population in Taiwan (Hwang and Chan 2002). The records contained data of the medical institutions' iden- 
tification, township names, date-of-visit, patient's identification, gender, date of birth, code for emergency visit, and code for the discharge diagnosis by the International Classification of Diseases, Ninth Revision (ICD-9). The ER visit records were claimed by 85 hospitals and clinics with emergency medical service in Taipei City during the period January 1, 1997, to December 31, 1998. The cumulative distribution of the patients was $48.3 \%, 72.2 \%$ and $94.8 \%$ from the largest 10, 20 and 40 hospitals. People with minor illness may choose emergency service because of medical accessibility and other reasons. Therefore, to eliminate possible bias, we excluded patients whose medical expense for the visit paid by BNHI was less than the $5^{\text {th }}$ percentile of medical expenses of the recorded patients. The patients who had no additional clinic visits in Taipei City during the study period were also excluded from the dataset because those patients might not live in the city. Separate daily counts were assembled for the discharge diagnosis from respiratory diseases of acute bronchitis and bronchiolitis, pneumonia and influenza, chronic bronchitis, emphysema, and asthma (ICD-9 codes 466, 480-493), cardiac diseases of ischemic heart disease, cardiac dysrhythmias, and heart failure (ICD-9 codes 410-414, 427-428) and gastrointestinal illness of gastric ulcer, duodenal ulcer, and peptic ulcer (ICD-9 codes 531-533). We further classified these three disease counts series into 3 age strata: children (0-14), adults (15-64) and the elderly $(65+)$, respectively, in order to evaluate age-specific pollution effects. Gastrointestinal illness was used as a sham outcome to check potential artificial pollution effects due to disease-biased hospital's admission practice and patient's access to medical service in our statistical models.

Table 1: Distribution of daily emergency room visits for respiratory, cardiac and gastrointestinal diseases by age strata in Taipei, Taiwan 1997-1998.

\begin{tabular}{crrrrrrr}
\hline & \multicolumn{3}{c}{ Respiratory } & \multicolumn{2}{c}{ Cardiac } & \multicolumn{2}{c}{ Gastrointestinal } \\
\cline { 2 - 7 } Percentile & $0-14$ & $15-64$ & $65+$ & $15-64$ & $65+$ & $15-64$ & $65+$ \\
\hline $10 \%$ & 14 & 8 & 7 & 5 & 10 & 6 & 2 \\
$25 \%$ & 18 & 10 & 10 & 7 & 14 & 8 & 4 \\
$50 \%$ & 25 & 14 & 14 & 9 & 17 & 10 & 6 \\
$75 \%$ & 34 & 19 & 20 & 12 & 21 & 13 & 8 \\
$90 \%$ & 48 & 24 & 28 & 15 & 25 & 16 & 10 \\
Mean & 29 & 15 & 17 & 10 & 18 & 11 & 6 \\
\hline
\end{tabular}

The distributions of age-specific emergency room visits for respiratory, cardiac and gastrointestinal diseases between January 1997 and December 1998 in Taipei City are shown in Table 1. Mean daily ER visits were 15-29 for respiratory diseases, 10-18 for cardiac diseases, and 6-11 for gastrointestinal diseases during 
the study period. The young had the most ER visits for respiratory diseases and the elderly had the most ER visits for cardiac diseases. The number of adult ER visits for gastrointestinal diseases were higher than that of the elderly.

\subsection{Air pollution and weather data}

The five air quality monitoring stations in Taipei measured hourly levels of $\mathrm{SO}_{2}, \mathrm{NO}_{2}, \mathrm{CO}, \mathrm{PM}_{10}$ and $\mathrm{O}_{3}$ since September, 1994. One of these five stations also measured $\mathrm{PM}_{2.5}$ since April 16, 1997. We obtained 24-hour average for $\mathrm{NO}_{2}$, $\mathrm{SO}_{2}, \mathrm{PM}_{2.5}$ and $\mathrm{PM}_{10}$, hourly maximum $\mathrm{O}_{3}$, and maximum 8-hour running average for $\mathrm{CO}$ from each monitoring station and averaged them over five ambient stations to represent the population's daily exposures to air pollutants. Daily meteorological conditions of wind direction, wind speed, temperature, dew point and precipitation were also averaged over the measurements in five monitoring stations. Daily maximum temperature and average dew point temperature were used to adjust the meteorological effects on ER visits. Note that $\mathrm{PM}_{2.5}$ measurements were available from one downtown station only.

Table 2: Summary statistics of environmental variables, in Taipei, Taiwan, 1997-1998.

\begin{tabular}{ccccccccc}
\hline Percentile & $\begin{array}{c}\mathrm{PM}_{10} \\
\left(\mu g / m^{3}\right)\end{array}$ & $\begin{array}{c}\mathrm{PM}_{2.5} \\
\left(\mu g / m^{3}\right)\end{array}$ & $\begin{array}{c}\mathrm{NO}_{2} \\
(\mathrm{ppb})\end{array}$ & $\begin{array}{c}\mathrm{SO}_{2} \\
(\mathrm{ppb})\end{array}$ & $\begin{array}{c}\mathrm{CO} \\
(\mathrm{ppm})\end{array}$ & $\begin{array}{c}\mathrm{O}_{3}^{*} \\
(\mathrm{ppb})\end{array}$ & $\begin{array}{c}\mathrm{TP}^{*} \\
\left({ }^{\circ} \mathrm{C}\right)\end{array}$ & $\begin{array}{c}\mathrm{DTP}^{*} \\
\left({ }^{\circ} \mathrm{C}\right)\end{array}$ \\
\hline $10 \%$ & 23.4 & 17.1 & 20.5 & 1.6 & 0.9 & 22.9 & 17.7 & 2.5 \\
$25 \%$ & 32.1 & 22.7 & 24.4 & 2.5 & 1.1 & 30.7 & 22.0 & 3.7 \\
$50 \%$ & 43.6 & 29.6 & 29.6 & 3.7 & 1.4 & 39.6 & 27.6 & 6.5 \\
$75 \%$ & 58.5 & 38.7 & 35.1 & 5.3 & 1.9 & 60.0 & 32.0 & 8.2 \\
$90 \%$ & 80.5 & 50.6 & 41.1 & 7.3 & 2.2 & 86.8 & 34.4 & 9.5 \\
Mean & 48.3 & 32.1 & 30.2 & 4.1 & 1.5 & 48.0 & 26.8 & 6.2 \\
\hline
\end{tabular}

* $\mathrm{O}_{3}$, daily maximum ozone concentration; $\mathrm{TP}$, daily maximum temperature; DTP, difference between daily maximum and minimum temperature.

Table 2 summarizes air pollution and weather data over the study period in Taipei. Mean daily concentrations of air pollutants for over two years were $48.3 \mu \mathrm{g} / \mathrm{m}^{3}$ for $\mathrm{PM}_{10}, 32.1 \mu \mathrm{g} / \mathrm{m}^{3}$ for $\mathrm{PM}_{2.5}, 30.2 \mathrm{ppb}$ for $\mathrm{NO}_{2}$, and $4.1 \mathrm{ppb}$ for $\mathrm{SO}_{2}$. The average of daily maximum 1-h ozone and 8-h CO concentrations were $48 \mathrm{ppb}$ and $1.5 \mathrm{ppm}$, respectively. The daily maximum temperatures (TP) averaged at $26.8^{\circ} \mathrm{C}$, and the differences between daily maximum and minimum temperatures (DTP) averaged at $6.2^{\circ} \mathrm{C}$. The data from 1997 to 1998 indicated that Taipei was a warm city with a relatively large difference between day and night temperatures, and polluted by high concentrations of $\mathrm{PM}, \mathrm{NO}_{2}$, and $\mathrm{O}_{3}$. 


\subsection{Statistical analysis}

Instead of using generalized additive models to fit the data, we adopted a cautious model construction procedure with simple implementation in most statistical software. To ensure that pollution effects were not confounded by trend, season, day of the week, and weather factors, the mean equation of the overdispersed Poisson model for an ER visit series $y_{t}$, was first given by

$$
\log \left[E\left(y_{t}\right)\right]=L_{t}+S_{t}+D_{t}+H_{t}+W_{t},
$$

where $E\left(y_{t}\right)$ is the expected number of the ER visits on the $t^{\text {th }}$ day; the component $L_{t}=\sum_{j=1}^{p} \phi_{j} \log \left(\max \left(y_{t-j}, 1\right)\right)$ is an explanatory variable of lagged values of the dependent variable; $S_{t}=\varphi_{1} \sin (4 t \pi / 365)+\varphi_{2} \cos (4 t \pi / 365)$ is a time series with a period of half year; $D_{t}$ consists of items representing days of the week; $H_{t}$ consists of items for special holidays such as the week-long Lunar New Year and some months with extreme weather, such as January, February, July and August; and $W_{t}$ consists of series of daily temperature difference, maximum temperature, temperature average of previous three days, dew point and rain fall. The variance of the dependent variable is assumed to be proportional to the expectation of the series.

The lagged component $L_{t}$ was added to remove the autocorrelation of the observed outcome series. The parametric time series of $S_{t}$ was added to model general temporal pattern of higher disease outcomes in the winter and summer, and $H_{t}$ removed effects due to special holidays and extreme weather. The time series $D_{t}$ removed differences in ER visits between days of the week. The explanatory variables were chosen to minimize Akaike's information criterion (AIC) in a stepwise procedure. The deviations in the expected number of ER visits of the selected model to the observed series were further examined for any autocorrelation, temporal and seasonal patterns. When the confounding variables were fixed, we separately added $\eta_{t}=\beta V_{t}$, where $V_{t}$ is the daily pollutant level lagged 0-3 days, to the mean equation of the selected model to complete a single-pollutant model, which is $\log \left[E\left(y_{t}\right)\right]=L_{t}+S_{t}+D_{t}+H_{t}+W_{t}+\eta_{t}$. The expected relative risk of ER visit for any individual on a day with a new pollution level, denoted by $V^{(1)}$, to a baseline pollution level, denoted by $V^{(0)}$, is $R R=E\left(y_{t} \mid V^{(1)}\right) / E\left(y_{t} \mid V^{(0)}\right)=\exp \left[\beta \times\left(V^{(1)}-V^{(0)}\right)\right]$. That is, the expected relative risk can be estimated by the exponential of the estimated pollution coefficient, $\beta$, for the added pollutant multiplied by the difference of the two pollutant levels. The $1^{\text {st }}$ quartile of measured pollution level is often treated as a baseline; while the $3^{\text {rd }}$ quartile of the pollution level is chosen as a risk level for comparing the relative risk.

The multi-pollutant model was constructed by replacing $\eta_{t}=\beta V_{t}$ in the single-pollutant model with $\eta_{t}=\sum_{i=1}^{p} \beta_{i} V_{i t}$, where $V_{1 t}, \cdots, V_{p t}$ are the daily 
levels of the $p$ pollutants with a specified time lag. To construct the mixture model, we simply modified the multi-pollutant model by adding extra terms representing the ratios of all pairs of pollutants considered. In this study, we added only those ratios of gaseous pollutants to the fine particulate matter. Let $V_{1 t}$ represent $\mathrm{PM}_{2.5}$ and $P_{2 t}, \cdots, P_{q t}$ represent the ratios of other gaseous pollutants to $V_{1 t}$, and then we have $\eta_{t}=\sum_{i=1}^{q} \beta_{i} V_{i t}+\sum_{i=2}^{q} \alpha_{i} P_{i t}$ in the final mixture model. With estimated coefficients $\hat{\alpha}_{i}, \hat{\beta}_{i}$ and estimated standard errors and correlations of these estimated coefficients from the final mixture models, we made a similar inference on relative risk increase on increments of air pollution mix. Let $V_{i}^{(0)}$ be a baseline level for the $i^{\text {th }}$ pollutant, $V_{i}^{(1)}$ be a new level of the pollutant and $V_{i}^{(d)}=V_{i}^{(1)}-V_{i}^{(0)}$. The ratios and ratio differences were denoted by $P_{i}^{(j)}=V_{i}^{(j)} / V_{1}^{(j)}$ and $P_{i}^{(d)}=P_{i}^{(1)}-P_{i}^{(0)}$, respectively. The relative risk is written as $R R=M \times A$, where $M=\exp \left[\sum_{i=2}^{q} \hat{\alpha}_{i} P_{i}^{(d)}\right]$ and $A=\exp \left[\sum_{i=1}^{q} \hat{\beta}_{i} V_{i}^{(d)}\right]$ representing relative risks of the blending effect and total amount effect of a new pollution mix relative to a baseline pollution mix, respectively. We interpret $\mathrm{A}$ as the expected relative risk contributed by the increase in total amount of a pollution mix. Note that $R R=A$ when we choose the multi-pollutant model. $M$ represents a blending effect of ratio changes in the pollution mix. The estimates of $95 \%$ confident intervals (CI) of the relative risks $R R, A$ and $M$ can be obtained straightforward. For example, the estimate of standard error of $\log (M)$, denoted by $S_{M}$, is given by the square root of $\sum_{i=2}^{q} \operatorname{var}\left(\hat{\alpha}_{i}\right) P_{i}^{(d)} P_{i}^{(d)}+2 \sum_{2 \leq i \leq j \leq q} \operatorname{cov}\left(\hat{\alpha_{i}}, \hat{\alpha_{j}}\right) P_{i}^{(d)} P_{j}^{(d)}$. The lower and upper bound of the $95 \% \mathrm{CI}$ is estimated by $\exp \left[M \pm 1.96 S_{M}\right]$. Note that the correlations of the ratios of gaseous pollutants to $\mathrm{PM}_{2.5}$ tend to be very high, since these pollutants are often correlated with $\mathrm{PM}_{2.5}$. Theoretically the regression model will produce large negative values of $\operatorname{cov}\left(\hat{\alpha_{i}}, \hat{\alpha_{j}}\right)$. Therefore, we expect small standard error estimates for relative risk estimates for the blending effect. The significance of $M$ will then affect the gain of the mixture model from the multi-pollutant model. We suggest that the judgment of significant difference between the multi-pollutant model and the proposed mixture model be determined by the deviances of the two models.

\section{Results}

The Pearson's correlation coefficients among 6 air pollutants and 2 weather parameters in Taipei are shown in Table 3 with the correlation of levels in the upper triangle. Daily $\mathrm{PM}_{10}$ and $\mathrm{PM}_{2.5}$ concentrations were highly correlated $(r=0.83)$. Daily $\mathrm{PM}_{10}$ and $\mathrm{PM}_{2.5}$ concentrations were moderately correlated with daily $\mathrm{NO}_{2}, \mathrm{SO}_{2}$, and $\mathrm{CO}(r=0.55-0.67)$. Daily $\mathrm{O}_{3}$ concentrations were correlated moderately with DPT $(r=0.61)$ and slightly with TP $(r=0.49)$. 
As shown in the lower triangle of Table 3, the correlation coefficients of daily ratios of the 4 gaseous pollutants to $\mathrm{PM}_{2.5}$ levels were, as expected, very high $(r=0.92-0.97)$.

Table 3: Pearson's correlation coefficients between air pollution and weather variables in Taipei, Taiwan, 1997-1998; The upper triangular was obtained based on daily levels of the 6 pollutants; while the 6 elements in the lower triangular were obtained based on daily ratios of 4 gaseous pollutants to $\mathrm{PM}_{2.5}$ levels.

\begin{tabular}{lcccccccc}
\hline & $\mathrm{PM}_{10}$ & $\mathrm{PM}_{2.5}$ & $\mathrm{NO}_{2}$ & $\mathrm{SO}_{2}$ & $\mathrm{CO}$ & $\mathrm{O}_{3}$ & $\mathrm{TP}$ & $\mathrm{DTP}$ \\
\hline $\mathrm{PM}_{10}$ & 1 & 0.83 & 0.66 & 0.67 & 0.55 & 0.47 & 0.14 & 0.43 \\
$\mathrm{PM}_{2.5}$ & & 1 & 0.67 & 0.64 & 0.56 & 0.43 & 0.00 & 0.39 \\
$\mathrm{NO}_{2}$ & & & 1 & 0.65 & 0.75 & 0.43 & -0.03 & 0.28 \\
$\mathrm{SO}_{2}$ & & & 0.94 & 1 & 0.55 & 0.52 & 0.33 & 0.51 \\
$\mathrm{CO}$ & & & 0.97 & 0.93 & 1 & 0.33 & 0.19 & 0.38 \\
$\mathrm{O}$ & & & 0.95 & 0.92 & 0.92 & 1 & 0.49 & 0.61 \\
$\mathrm{TP}$ & & & & & & & 1 & 0.72 \\
$\mathrm{DTP}$ & & & & & & & & 1 \\
\hline
\end{tabular}

We performed 72 single-pollutant models for respiratory diseases in three age strata, 48 models for cardiac diseases, and another 48 models for gastrointestinal diseases in two age strata separately. For each daily health outcome series, we used parametric models to remove temporal and seasonal patterns, day of the week and special holiday effects, and weather factors. Each final model was determined based on AIC and diagnostic plots of the residuals. The residual analysis included checking whether the confound effects and autocorrelation have been removed. We also checked boxplots of residuals in months, days of the week, special holiday versus regular days to ensure that all possible confounding effects were being adjusted. Before adding air pollution variables to each selected model, we plotted the residuals against each pollutant levels to see any possible linear and nonlinear pattern. As an example shown in Figure 1, the smoothed curve shows that there is a linear association between $\mathrm{PM}_{2.5}$ levels and the residuals. Hence, single pollutant term of levels of same day and previous 3 days was added to the mean equation of the Poisson regression model separately for the period April 16, 1997 - December 31, 1998. The estimated pollution coefficients were then used to calculate relative risks for an increase of the interquartile range for the pollutants in the study period.

Table 4 lists the significantly increased relative risks of ER visits due to respiratory diseases for an IQR increment in pollutant concentrations estimated by the single-pollutant Poisson regression models. Both particulate $\left(\mathrm{PM}_{2.5}\right.$ and $\left.\mathrm{PM}_{10}\right)$ 


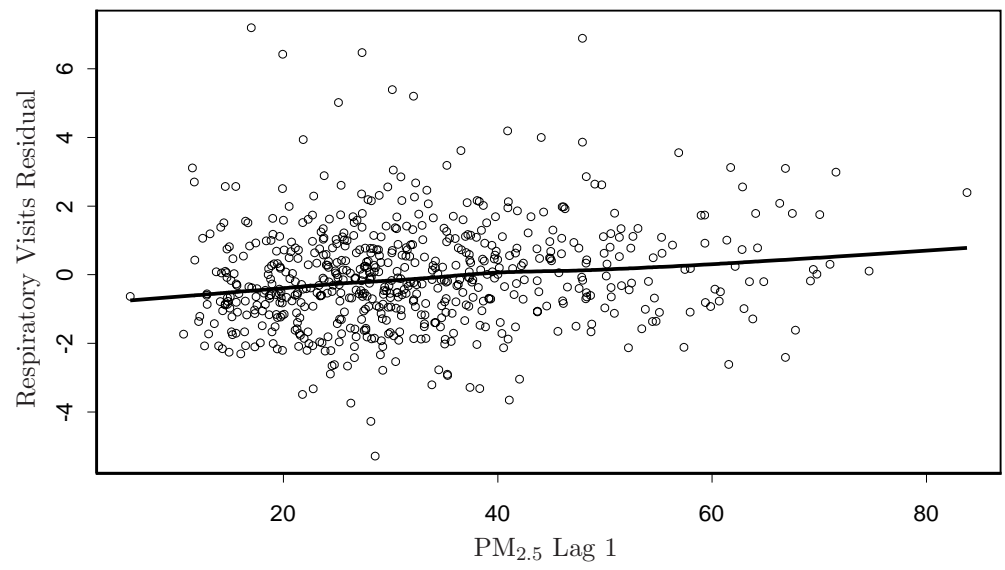

Figure 1: A plot of the residual counts of emergency room visits respiratory disease in 0-14 years of age group in Taipei, during 1997-1998 versus average $\mathrm{PM}_{2.5}$ levels of one-day lag during the study period. The residuals have been adjusted for all patterns and weather variables except air pollution in a Poisson model. The line is drawn using loess, a smoothing function in S-Plus statistical software, on the data.

and gaseous pollutants $\left(\mathrm{NO}_{2}, \mathrm{CO}\right.$, and $\left.\mathrm{O}_{3}\right)$ significantly increased children's ER visits for respiratory diseases. The relative risks of children's ER visits were about 1.04-1.06 for a $16 \mu \mathrm{g} / \mathrm{m}^{3}$ increment of $\mathrm{PM}_{2.5}$ at $0-3$ day lags. Estimated relative risks of other air pollutants were: $1.03-1.04$ for $\mathrm{PM}_{10}$ lagged 1-3 days $(95 \% \mathrm{CI}=$ 1.00 - 1.07; IQR $\left.=26.4 \mu \mathrm{g} / \mathrm{m}^{3}\right) ; 1.03-1.04$ for $\mathrm{NO}_{2}$ lagged $2-3$ days $(95 \% \mathrm{CI}=$ $1.00-1.07$; IQR $=10.7 \mathrm{ppb}) ; 1.04$ for $\mathrm{CO}$ lagged $2-3$ days $(95 \% \mathrm{CI}=1.00-1.08$; $\mathrm{IQR}=0.8 \mathrm{ppm}) ; 1.04$ for $\mathrm{O}_{3}$ lagged 2 days $(95 \% \mathrm{CI}=1.01-1.07 ; \mathrm{IQR}=29.3$ $\mathrm{ppb})$. For adults and the elderly, only particulate pollutants affected their ER visits for respiratory diseases. The relative risks were 1.04 at an IQR increment in 2-day lagged $\mathrm{PM}_{2.5}$ and 3-day lagged $\mathrm{PM}_{10}$ for adults, and was 1.04-1.05 for $\mathrm{PM}_{2.5}$ lagged 0-3 days and 1.03 for 2-day lagged $\mathrm{PM}_{10}$ per IQR increment.

Table 5 lists the significantly increased relative risks of ER visits due to cardiac diseases for an IQR increment in pollutant concentrations estimated by the single-pollutant Poisson regression models. We observed that the pollution effects occurred mostly at current-day exposures for adults and at 2-3 days lagged exposures for the elderly. The estimated relative risks of adults' cardiac ER visits were 1.05, 1.06 and 1.06 per IQR increment of their current-day exposures to $\mathrm{PM}_{2.5}, \mathrm{NO}_{2}$ and $\mathrm{CO}$, respectively. For the elderly, their relative risks associated 
Table 4: Estimated relative risk in emergency room visits and 95\% CIs for an IQR increase in pollutants from single-pollutant lagged Poisson models for respiratory disease in Taipei City, Taiwan, 1997-1998.

\begin{tabular}{ccccc}
\hline Age & Pollutant & Lag & Relative Risk & $95 \%$ CI \\
\hline Children & $\mathrm{PM}_{2.5}$ & 0 & 1.039 & $(1.007,1.070)$ \\
& & 1 & 1.057 & $(1.027,1.088)$ \\
& & 2 & 1.051 & $(1.019,1.082)$ \\
& $\mathrm{PM}_{10}$ & 1 & 1.036 & $(1.005,1.068)$ \\
& & 2 & 1.038 & $(1.004,1.059)$ \\
& & 3 & 1.028 & $(1.001,1.052)$ \\
& $\mathrm{NO}_{2}$ & 2 & 1.041 & $(1.010,1.072)$ \\
& & 3 & 1.033 & $(1.002,1.064)$ \\
& $\mathrm{CO}$ & 2 & 1.043 & $(1.007,1.079)$ \\
& & 3 & 1.038 & $(1.002,1.074)$ \\
Adults & $\mathrm{O}_{3}$ & 2 & 1.040 & $(1.010,1.070)$ \\
\hline Elderly & $\mathrm{PM}_{2.5}$ & 2 & 1.037 & $(1.001,1.073)$ \\
& $\mathrm{PM}_{10}$ & 3 & 1.036 & $(1.006,1.065)$ \\
\hline & $\mathrm{PM}_{2.5}$ & 0 & 1.046 & $(1.009,1.082)$ \\
& & 1 & 1.042 & $(1.009,1.075)$ \\
& & 2 & 1.035 & $(1.002,1.068)$ \\
& & 3 & 1.043 & $(1.010,1.076)$ \\
& $\mathrm{PM}_{10}$ & 2 & 1.027 & $(1.001,1.054)$ \\
\hline
\end{tabular}

with $\mathrm{PM}_{2.5}$ and $\mathrm{PM}_{10}$ at lagged 2-3 days were about 1.05 and 1.03 per IQR increment, respectively. The relative risks of cardiac ER visits were 1.04 for $\mathrm{CO}$ lagged 1 day, 1.04 for $\mathrm{SO}_{2}$ lagged 2 days and $\mathrm{NO}_{2}$ lagged 3 days among the elderly. Among all these particulate and gaseous pollutants, $\mathrm{PM}_{2.5}$ was the most consistent air pollutant responsible for increase in daily ER visits for both respiratory and cardiac diseases. By contrast, none of these air pollutants had effect on daily ER visits for gastrointestinal diseases. It assured the fact that the estimated pollution effects on the cardiorespiratory disease have little bias due to hospital's admission practice and patient's access to medical service in our statistical models.

The results of single-pollutant models indicated that ER visits for respiratory diseases among children were affected by particulate and gaseous pollutants at 2-3 day lags during the study period. As to the ER visits for cardiac diseases, current-day air pollution mix also affected adults and air pollution mix with 2-3 day lags affected the elderly. For comparison of modeling multiple pollutants, we 
considered mixtures of $\mathrm{PM}_{2.5}$ and gaseous pollutants $\mathrm{NO}_{2}, \mathrm{SO}_{2}, \mathrm{CO}$ and $\mathrm{O}_{3}$ on the five specific scenarios listed in Table 6 . The results show that the relative risks of ER visits for respiratory disease in the 0-14 years of age group were 1.069 $(95 \% \mathrm{CI}=1.025-1.114)$ per IQR increment of a mix of $\mathrm{PM}_{2.5}, \mathrm{NO}_{2}, \mathrm{CO}$ and $\mathrm{O}_{3}$ at lag 2 days using multi-pollutant model. The mixture model was significantly better fitted than the multi-pollutant model on this scenario. The joint effect $(R R=1.088 ; 95 \% \mathrm{CI}=1.041-1.136)$ was much higher than the effect of each single pollutant as shown in Figure 2. The effect of a mix of $\mathrm{PM}_{2.5}, \mathrm{NO}_{2}$ and $\mathrm{CO}$ at lag 3 days on respiratory disease in the children age group was relatively smaller $(R R=1.048)$, which was a little increase from the single pollutant effect.

Table 5: Estimated relative risks in emergency room visits and 95\% CIs for an IQR increase in pollutants from single-pollutant lagged Poisson models for cardiac disease in Taipei City, Taiwan, 1997-1998.

\begin{tabular}{ccccc}
\hline Age & Pollutant & Lag & Relative Risk & $95 \% \mathrm{CI}$ \\
\hline Adults & $\mathrm{PM}_{2.5}$ & 0 & 1.051 & $(1.007,1.098)$ \\
& $\mathrm{NO}_{2}$ & 0 & 1.058 & $(1.016,1.102)$ \\
& $\mathrm{CO}$ & 0 & 1.061 & $(1.012,1.112)$ \\
\hline Elderly & $\mathrm{PM}_{2.5}$ & 2 & 1.048 & $(1.017,1.079)$ \\
& & 3 & 1.045 & $(1.015,1.077)$ \\
& $\mathrm{PM}_{10}$ & 2 & 1.040 & $(1.014,1.066)$ \\
& & 3 & 1.034 & $(1.008,1.061)$ \\
& $\mathrm{NO}_{2}$ & 3 & 1.042 & $(1.014,1.072)$ \\
& $\mathrm{SO}_{2}$ & 2 & 1.037 & $(1.010,1.066)$ \\
& $\mathrm{CO}$ & 1 & 1.044 & $(1.010,1.078)$ \\
\hline
\end{tabular}

Table 6 and Figure 3 show that the fitted mixture model was significantly better than the multi-pollutant model in the case of mix of $\mathrm{PM}_{2.5}, \mathrm{NO}_{2}$ and $\mathrm{CO}$ at current day on ER visits for cardiac disease in the 15-64 years of age group. The estimated relative risk was $1.086(95 \% \mathrm{CI}=1.025$ - 1.151$)$ per IQR increment of a mix of $\mathrm{PM}_{2.5}, \mathrm{NO}_{2}$ and $\mathrm{CO}$ at current day using the mixture model. It indicates that there was blending effect of $\mathrm{PM}_{2.5}$ and gaseous pollutants on the ER visits for cardiac diseases in the 15-64 years of age group. While the two models had no difference in fitting the cardiac diseases in the elderly age group for exposure to mix of $\mathrm{PM}_{2.5}$ and gaseous pollutants. The relative risks were about 1.06 per IQR increment of the mixtures of pollutants. 
Respiratory disease (children)

Respiratory disease (children)
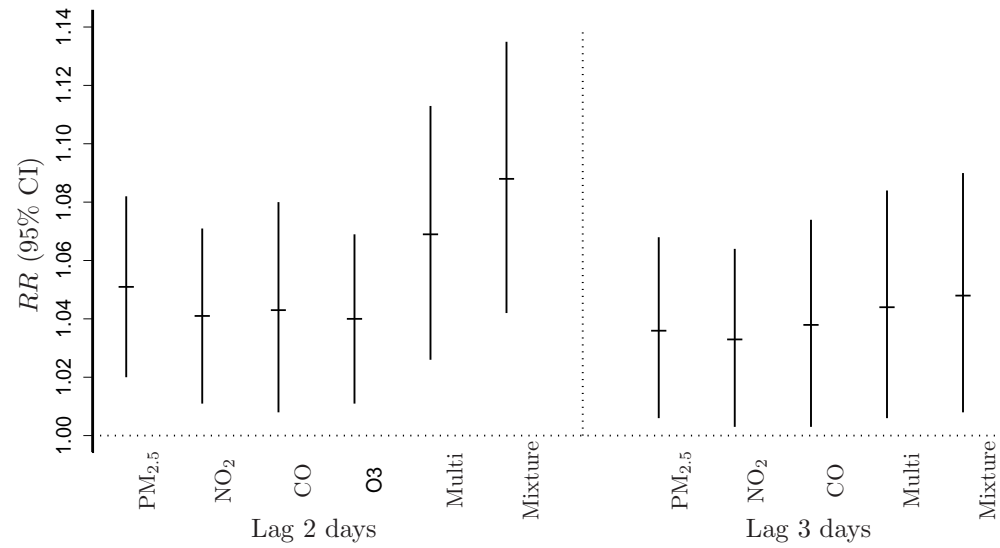

Figure 2: The effects of single pollutant and mix of pollutants on ER visit for respiratory disease in Taipei, 1997-1998, which are shown by the estimated relative risks and 95\% CIs for an IQR increase in pollutant levels. The estimates were obtained by single-pollutant, multi-pollutant and mixture models, respectively.

Table 6: Gaseous pollutants and $\mathrm{PM}_{2.5}$ mix effects on ER visits for respiratory and cardiac diseases. The values shown are model deviances, estimated relative risks and $95 \%$ confidence intervals (CI) for IQR changes in concentrations of the pollutants in multi-pollutant and mixture models.

\begin{tabular}{lccccc}
\hline $\begin{array}{l}\text { Disease, Age group, } \\
\text { Gaseous pollutants }\end{array}$ & Model & Deviance & P-value & $\begin{array}{c}\text { Relative } \\
\text { risk }\end{array}$ & 95\% CI \\
\hline Respiratory, 0-14 years, & Multi- & 1393.0 & 0.000 & 1.069 & $(1.025,1.114)$ \\
$\mathrm{NO}_{2}, \mathrm{CO}$ and $\mathrm{O}_{3}$ lag 2 days & Mixture & 1369.9 & & 1.088 & $(1.041,1.136)$ \\
Respiratory, 0-14 years, & Multi- & 1405.5 & 0.030 & 1.044 & $(1.005,1.085)$ \\
$\mathrm{NO}_{2}$ and CO lag 3 days & Mixture & 1398.5 & & 1.048 & $(1.007,1.091)$ \\
$\mathrm{Cardiac}^{15-64}$ years, & Multi- & 827.6 & 0.036 & 1.066 & $(1.009,1.127)$ \\
$\mathrm{NO}_{2}$ and CO current day & Mixture & 821.0 & & 1.086 & $(1.025,1.151)$ \\
$\mathrm{Cardiac}^{6}$ 65+ years, & Multi- & 803.2 & 0.438 & 1.058 & $(1.024,1.093)$ \\
$\mathrm{SO}_{2}$ lag 2 days & Mixture & 802.7 & & 1.060 & $(1.025,1.096)$ \\
$\mathrm{Cardiac}$ 65+ years, & Multi- & 803.3 & 0.877 & 1.060 & $(1.021,1.102)$ \\
$\mathrm{NO}_{2}$ and CO lag 3 days & Mixture & 802.9 & & 1.062 & $(1.021,1.105)$ \\
\hline
\end{tabular}

\section{Discussion}

Our study shows that there were significant associations between air pollution 
and daily ER visits for respiratory and cardiac diseases, but not for gastrointestinal illness in Taipei City during the period 1997-1998. $\mathrm{PM}_{2.5}$ was the most consistent single pollutant related significantly to daily ER visits for both respiratory and cardiac diseases. $\mathrm{PM}_{10}$ showed weaker effects on daily ER visits for respiratory and cardiac diseases in the single-pollutant model and was not as consistent as $\mathrm{PM}_{2.5}$. Although gaseous air pollutants had some effects on ER visits, such as $\mathrm{NO}_{2}, \mathrm{CO}$ and $\mathrm{O}_{3}$ on respiratory diseases, and $\mathrm{CO}, \mathrm{NO}_{2}$ and $\mathrm{SO}_{2}$ on cardiac diseases, their individual contributions to relative risks were comparatively lower than that of $\mathrm{PM}_{2.5}$.

This study provides epidemiologic evidence that fine particles in urban environments are a risk factor for cardiopulmonary diseases. Respiratory epithelium can be activated by exposure to fine particles containing various combustion products including transition metals and organics from gasoline and diesel powered automobile emissions. One recent in-vitro study has found significant cellular toxicity induced by fine particles and $\mathrm{O}_{3}$ in Taipei (Huang et al. 2003). A growing number of panel studies have reported that the PM's effects on cardiovascular morbidity are possible through either coagulation in blood vessel or changes in heart rate modulation induced by pulmonary inflammation (Ibald-Muli et al. 2001; Magari et al. 2001). One recent animal toxicity study has shown concentrated ambient particles in Taipei significantly induced heart rates and blood pressures of pulmonary hypertensive rats (Cheng et al. 2003). Although we still lack complete understanding about the biological plausibility of PM toxicity, our findings demonstrate that the effect of PM on cardiopulmonary morbidity are beyond western experience and might be a worldwide phenomenon.

Although multi-pollutant models have been proposed for modeling health effect of multiple pollutants in several studies, the models were used only for estimating single pollutant effect with an adjustment of other pollutants. In this study, we generalized the multi-pollutant model to the mixture model and used it for examining the joint effect of $\mathrm{PM}_{2.5}$ in combination with gaseous pollutants. We found that the risks of ER visits increased to $8.8 \%$ for respiratory disease in the children age group, $8.6 \%$ and $6 \%$ for cardiac disease in the adults and elderly age groups for an increment of interquartile range of pollution mixes in Taipei City, respectively. The effects of pollution mix were greater than the $\mathrm{PM}_{2.5}$ effects alone, which were about $4-5 \%$, but less than the sum of individual pollutant effects in the mixture.

Although the use of nonparametric smooth functions for the confounders was popular in most recent studies, we found it was sensitive to the choice of smoothing parameters. Therefore, we built our models through intensive data analysis step by step. In each step, a parametric model was constructed to fit the found pattern from data analysis and residuals were checked against each independent 


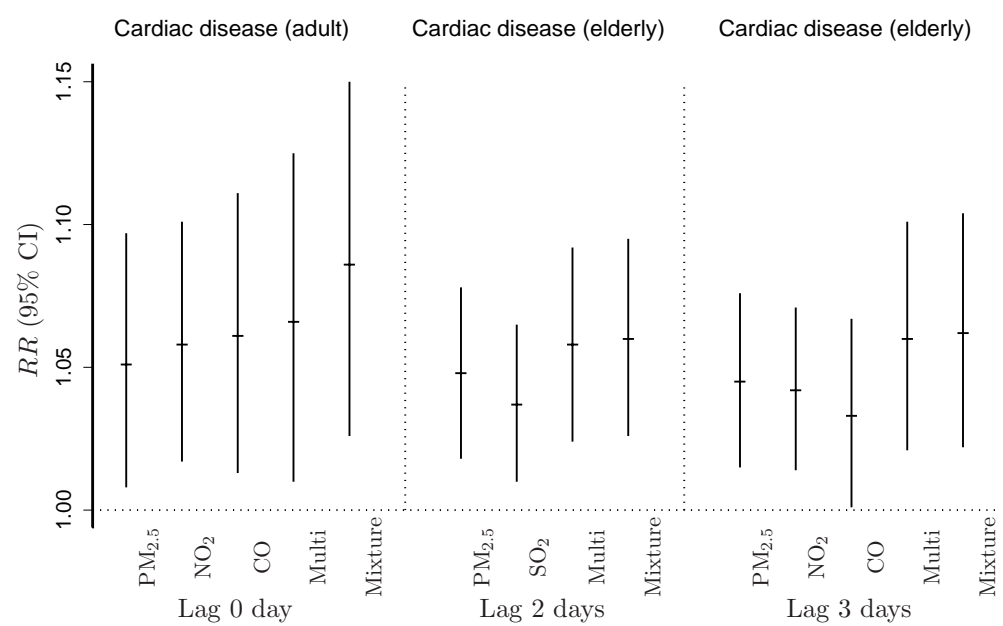

Figure 3: The effects of single pollutant and mix of pollutants on ER visit for cardiac disease in Taipei, 1997-1998, which are shown by the estimated relative risks and 95\% CIs for an IQR increase in pollutant levels. The estimates were obtained by single-pollutant, multi-pollutant and mixture models, respectively.

variable to ensure that the confounding effects had been removed sufficiently. The autocorrelation of outcome series was removed by including lagged dependant variables as independent variables rather than by modeling the error as an autoregressive process. Accordingly, the computation became much simpler by using regularly available Poisson regression programs in any statistical software. We believed this analytical approach would improve our estimation of air pollution effects in population-based epidemiologic studies, which were usually inherent with many locally confounded factors.

\section{Acknowledgments}

We greatly appreciate Taiwan Environmental Protection Administration and Bureau of National Health Insurance in providing the data. The work was partially supported by the Academia Sinica grant AS-89-TP-A09.

\section{References}

Burnett, R., Dales, R., Krewski, D., Vincent, R., Dann, T. and Brook, J. (1995). Associations between ambient particulate sulfate and admissions to Ontario hospitals for cardiac and respiratory diseases. American Journal of Epidemiology 142, 15-22. 
Burnett, R., Cakmak, S., Brook, J. and Krewski, D. (1997). The role of particulate size and chemistry in the association between summertime ambient air pollution and hospitalization for cardiorespiratory diseases. Environmental Health Perspectives 105, 614-620.

Burnett, R., Smith-Doiron, M., Stieb, D., Raizenne, M., Brook, J., Dales, R., Leech, J., Cakmak, S. and Krewski, D. (2001). Association between ozone and hospitalization for acute respiratory diseases in children less than 2 years of age. American Journal of Epidemiology 153, 444-452.

Cheng, T. J., Hwang, J. S., Wang, P. W. et al. (2003). Effects of concentrated ambient particles on heart rate and blood pressure in pulmonary hypertensive rats. Environmental Health Perspectives 111, 147-150.

Delfino, R., Murphy-Moulton, A. and Becklake, M. (1998). Emergency room visits for respiratory illness among the elderly in Montreal: Association with low level ozone exposure. Environmental Research Section A 76, 67-77.

Huang, S. L., Hsu, M. and Chan, C. C. (2003). Effects of submicronmeter particle's compositions on cytokine production and lipid peroxidation of human bronchial epithelial cells. Environmental Health Perspectives 111, 478-482.

Hwang, J. S. and Chan, C. C. (2002). Effects of air pollution on daily clinic visits for lower respiratory tract illness (with commentary). American Journal of Epidemiology 155, 1-16.

Hwang, J. S. and Chen, J. (1999). An evaluation of risk estimation procedures for mixtures of carcinogens. Risk Analysis 19, 1071-1076.

Ibald-Muli, A., Stieber, J., Wichmann, H. E. et al. (2001). Effects of air pollution on blood pressure: a population-based approach. American Journal of Public Health 91, 571-577.

Linn, W., Szlachcic, Y., Gong, H., Kinney, P. and Berhane, K. (2000). Air pollution and daily hospital admissions in metropolitan Los Angeles. Environmental Health Perspectives 108, 427-434.

Lipsett, M., Hurley, S. and Ostro, B. (1997). Air pollution and emergency room visits for asthma in Santa Clara County, California. Environmental Health Perspectives 105, 216-222.

Magari, S. R., Hauser, R., Schwartz, J. et al. (2001). Association of heart rate variability with occupational and environmental exposure to particulate air pollution. Circulation 104, 986-991.

Moolgavkar, S., Luebeck, E. and Anderson, E. (1997). Air pollution and hospital admissions for respiratory causes in Minneapolis-St. Paul and Birmingham. Epidemiology 8, 364-370.

Norris, G., YongPong, S., Koenig, J., Larson, T., Sheppard, L. and Stout, J. (1999). An association between fine particles and asthma emergency department visits for children in Seattle. Environmental Health Perspectives 107, 489-493. 
Schwartz, J. and Morris, R. (1995). Air pollution and hospital admission for cardiovascular disease in Detroit, Michigan. American Journal of Epidemiology 142, 23-35.

Schwartz, J. (1996). Air pollution and hospital admissions for respiratory disease. Epidemiology 7, 20-28.

Schwartz, J. (1997). Air pollution and hospital admissions for cardiovascular disease in Tucson. Epidemiology 8, 371-377.

Schwartz, J. (1999). Air pollution and hospital admissions for heart disease in eight U.S. counties. Epidemiology 10, 17-22.

Schwartz, J. (2000). Harvesting and long term exposure effects in relation between air pollution and mortality. American Journal of Epidemiology 151, 440-448.

Sheppard, L., Levy, D., Norris, G., Larson, T. and Koenig, J. (1999). Effects of ambient air pollution on nonelderly asthma hospital admissions in Seattle, Washington, 1987-1994. Epidemiology 10, 23-30.

Sunyer, J., Saez, M., Murillo, C., Castellsague, J., Martinez, F. and Anto J. M. (1993). Air pollution and emergency room admissions for chronic obstructive pulmonary disease: A 5-year study. American Journal of Epidemiology 137, 701-705.

Wong, C., Atkinson, R., Anderson, H., Hedley, A., Ma, S., Chau, P. and Lam, T. (2002). A tale of two cities: Effects of air pollution on hospital admissions in Hong Kong and London compared. Environmental Health Perspectives 110, 67-77.

Xu, X., Li, B. and Huang, H. (1995). Air pollution and unscheduled hospital outpatient and emergency room visits. Environmental Health Perspectives 103, 286-289.

Zhang, J., Hu, W., Wei, F., Wu, G., Korn, L. and Chapman, R. (2002). Children's respiratory morbidity prevalence in relation to air pollution in four Chinese cities. Environmental Health Perspectives 110, 961-967.

Received February 13, 2004; accepted June 1, 2003.

\author{
Jing-Shiang Hwang \\ Institute of Statistical Science \\ Academia Sinica \\ Taipei, Taiwan \\ hwang@sinica.edu.tw \\ Tsuey-Hwa Hu \\ Institute of Statistical Science \\ Academia Sinica \\ Taipei, Taiwan \\ jshwang6@stat.sinica.edu.tw \\ Chang-Chuan Chan \\ Institute of Occupational Medicine and Industrial Hygiene
}


National Taiwan University ccchan@ha.mc.ntu.edu.tw 